\title{
TITLE:
}

\section{Heights of simple loops and pseudo-Anosov homeomorphisms}

$\operatorname{AUTHOR}(\mathrm{S})$ :

Kobayashi, Tsuyoshi

\section{CITATION:}

Kobayashi, Tsuyoshi. Heights of simple loops and pseudo-Anosov homeomorphisms. 数 理解析研究所講究録 1987, 624: 170-193

ISSUE DATE:

1987-05

URL:

http://hdl.handle.net/2433/99924

RIGHT: 
Heights of simple loops and pseudo-Anosov homeomorphisms

\author{
(陌大理 7. 林家设) \\ Tsuyoshi Kobayashi
}

\title{
1. Introduction
}

Let $H$ be a 3 -dimensional, orientable handlebody of genus $g$ $(>1)$ and $\ell(C \partial H)$ be a connected essential simple closed curve. A simple loop is the ambient isotopy class of $l$ and we often abbreviate it by denoting $l$. A surface is a connected 2-manifold. Let $F$ be a 2 -sided surface properly embedded in a 3 -manifold $M$. F is essential if it is incompressible and not parallel to a subsurface of $\partial M$. Then we define the height of $\ell, h(l)$, as follows: $h(l)=\min \{-\chi(F) \mid(F, \partial F)(C(H, \partial H-l))$ is an essential surface, where $\chi(F)$ denotes the Euler characteristic of $F$.

In section 2 we see that $h(l)$ can be defined.

Fix a hyperbolic metric on $\partial H$. Let $L(C \partial H)$ be a geodesic lamination. We say that $L$ is of full type if there is a system of mutually disjoint incompressible disks $\left\{D_{1}, \ldots, D_{3 g-3}\right\}$ in $H$ such that $\partial D_{1} \cup \ldots \cup \partial D_{3 g-3}$ cuts $\partial H$ into $2 g-2$ pants $P_{1}, \ldots, P_{2 g-2}$, which satisfies:

(i) $V \partial D_{i}$ and $L$ intersect transversely and there is no 2-gon $B$ in $\partial H$ such that $\partial B=\alpha \cup B$ where $\alpha$ is a subarc of $\partial D_{1} \cup \ldots \cup \partial D_{3 g-3}, B$ is a subarc of $L$ and

(ii) for each $P_{i}$ we have;

for each pair of boundary components of $P_{i}$, there is asubarc of $L$ properly embedded in $P_{i}$, which joins the components.

Then the first result of this paper is; 
Theorem 1. Let $\mathrm{f}: \partial \mathrm{H} \rightarrow \partial \mathrm{H}$ be a pseudo-Anosov homeomorphism and $\ell(\mathrm{C \partial H})$ a simple loop. Suppose that the stable lamination of $f$ is of full type. Then $\lim _{\mathrm{p} \rightarrow \infty} \mathrm{h}\left(\mathrm{f}^{\mathrm{p}}(\ell)\right)=\infty$, i.e. for each $\mathrm{n}(z-1)$ there exists a constant $\mathrm{N}$ such that if $\mathrm{m}>\mathrm{N}$ then $\mathrm{h}\left(\mathrm{f}^{\mathrm{m}}(\ell)\right)>\mathrm{n}$.

We note that the assumption on the stable lamination of $f$ is essential. In fact Fathi-Laudenbach [4] showed that there exists a pseudo-Anosov homeomorphism $\varphi: \partial H \rightarrow \partial H$ which extends to a homeomorphism of $H$. Then clearly we have $h\left(\varphi^{n}(\ell)\right)=h(\ell)$, for each $\ell$ and $n$.

Let $f: \partial H \rightarrow \partial H$ be a homeomorphism and $D_{1} \cup \ldots \cup D_{g}$ a union of mutually disjoint incompressible disks in $H$ such that $D_{1} U \ldots U D_{g}$ cuts $H$ into a 3-cell. Then we get a compact 3-manifold whose boundary is a sphere by attaching 2-handles to $H$ along the simple loops $f\left(\partial D_{1}\right), \ldots, f\left(\partial D_{g}\right)$. We note that the obtained manifold does not depend on the choice of $D_{1}, \ldots, D_{g}$ (Lemma 4.1). Hence we denote the manifold by $\bar{M}_{f}$. Then $M_{f}$ denotes the manifold obtained from $\bar{M}_{f}$ by capping off the boundary by a 3 -cell. Roughly speaking, $M_{f}$ is obtained from $H$ by attaching a copy of $H$ by $f$.

As an application of Theorem 1, we have;

Theorem 2. Let $\mathrm{f}: \partial \mathrm{H} \rightarrow \partial \mathrm{H}$ be a pseudo-Anosov homeomorphism. Suppose that the invariant laminations of $f$ are of full type. Then. for each $\mathrm{n}(\mathbf{2 0})$, there is a constant $\mathrm{N}$ such that if $\mathrm{m}>\mathrm{N}$, then ${ }_{\mathrm{f}}^{\mathrm{m}}$ does not contain a 2-sided incompressible surface whose genus is less 
than or equal to $\mathrm{n}$.

\section{Preliminaries}

For the definitions of the standard terms in the $3-d i m e n s i o n a l$ topology we refer to $[6,7]$. We assume that the reader is familiar with $[1]$.

Let $H, \ell$ be as in section 1 . First we will show that the height of $\ell$ can be defined.

Lemma 2.1. There exists a 2-sided non-separating surface $\mathrm{S}$ properly embedded in ( $\mathrm{H}, \partial \mathrm{H}-\mathrm{l})$.

Proof. Let $M$ be the 3-manifold obtained from $H$ by at taching a 2-handle $D^{2} \times I$ along $\ell$. Since the genus of $H$ is greater than 1, the first Betti number of $M$ is greater than 0 . Hence by [6,Lemma 6.6], M contains a properly embedded, 2-sided, non-separating incompressible surface $S^{\prime}$. Then, by moving $S^{\prime}$ by an ambient isotopy, we may suppose that $S^{\prime}$ intersects the 2 -handle in horizontal disks. Hence $S=S^{\prime}-I n t\left(S^{\prime} \cap\left(D^{2} \times I\right)\right)$ is a $2-s^{\prime}$ ded non-separating surface properly embedded in $H$. Moreover, by moving $S$ by a tiny isotopy, we may suppose that $S$ is properly embedded in $(H, \partial H-\ell)$.

This completes the proof of Lemma 2.1 .

Then we have;

Corollary. There exists an essential surface properly embedded 
in $(\mathrm{H}, \partial \mathrm{H}-\ell)$.

Proof. Let $S$ be the surface obtained in Lemma 2.1. If necessary, by applying the loop theorem and performing a surgery on $S$, we may suppose that $S$ is incompressible in $H$. Since $S$ is non-separating, $S$ is not parallel to a subsurface in $\partial H$. Hence $S$ is essential.

Let $F$ be a closed, orientable surface of genus $g(>1)$ with a hyperbolic metric and PL(F) the space of projective measured laminations of $F$ with an appropriate topology. Then PL(F) is homeomorphic to the $6 \mathrm{~g}-7$ dimensional sphere $\mathrm{s}^{6 \mathrm{~g}-7}$. A simple loop is the ambient isotopy class of a closed, connected, 1-submanifold of F which is not contractible in F. Then it is known that the set of all simple loops together with the counting measure consist a dense subset of PL(F). Let $\ell$ be a simple loop. $\mathscr{G}_{\ell}$ (CPL(F)) denotes the set of all simple loops which are disjoint from $\ell$. Then let $\varphi(\ell ; 1)$ $=\mathscr{\varphi}_{\ell}$, and $\mathscr{\varphi}(\ell ; k)=\bigcup_{\ell \cdot \in \mathscr{\varphi}(\ell ; k-1)} \mathscr{\varphi}_{\ell \cdot(k>1)}$.

In this section we will prove;

Proposition 2.2. Let $\mathrm{f}: \mathrm{F} \rightarrow \mathrm{F}$ be a pseudo-Anosov homeomorphism with an unstable lamination $\mathrm{L}^{-}$and $l$ a simple loop. Then there exists a sequence of neighborhoods of $\mathrm{L}^{-}$in $\mathrm{PL}(\mathrm{F}),\left\{\mathrm{U}_{\mathrm{i}}\right\}_{\mathrm{i}=1}^{\infty}$, such that $U_{1} \supset U_{2} \supset \ldots$, and $U_{k} \cap \varphi(\ell ; k)=\phi$, for each $\mathrm{k}$.

Let $\tau$ (CF) be a maximal train track, i.e. the closure of each 
component of $F-\tau$ is a $3-g o n$. Then the set of all non-negative weights on $\tau$ defines a $6 g-7$ dimensional ball $B_{\tau}$ in PL(F). And the set of all positive weights on $\tau$ corresponds to the interior of $B_{\tau}[1,10]$.

Lemma 2.3. If $\ell$ is carried by $\tau$ with all weights positive, then ${ }^{\varphi}{ }_{\ell} \subset \mathrm{B}_{\tau}$.

Proof. Let $N(\tau)$ be a standard neighborhood of $\tau$. Since $\ell$ [Figure 1]

is carried by $\tau$, we may suppose that $\ell \subset N(\tau)$ and $\ell$ is transverse to the fibers. Let ${ }^{m \in \mathscr{S}_{\ell}}$. Since each component of $F-N(\tau)$ is contractible, we can isotope $m$ into $N(\tau)$ with $m \cap l=\phi$. If $m$ is transverse to the fibers of $N(\tau)$, then $m \in B_{\tau}$. Hence we suppose that $m$ is not transverse to the fibers. We can isotope $m$ so that $m$ is transverse to the fibers except neighborhoods of the switches which are unions of fibers. See Figure 2. Then the neighborhood of [Figure 2]

a switch will look like as in Figure 3 . We note that the subarcs of [Figure 3]

$m$ as $a_{1}$ or $a_{2}$ in Figure 3 play a bad role in this situation. Then we will show that we can eliminate such arcs by an isotopy. Let $\Delta$ be the closure of a component of $F-N(\tau)$ and $e$ an edge of the triangle $\Delta$. Then we have;

Assertion. There is a rectangle $R$ in $F$ such that $R \subset N(\tau)$, Int $R \cap \ell=\phi$, one edge of $R$ is a subarc of $\ell$, one edge of $R$ is 
$e$, and the rest edges of $R$ are subarcs of two fibers of $N(\tau)$.

We note that the universal cover of $F$ is isometric to the hyperbolic plane $\mathbb{H}^{2}$. Let $\widetilde{\Delta}\left(\mathrm{CH}^{2}\right)$ be a lift of $\Delta$, $\widetilde{e}$ the edge of $\widetilde{\Delta}$ [Figure 4]

corresponding to e and $\widetilde{N}(\tau)\left(\subset H^{2}\right)$ the lift of $N(\tau)$. Let $I_{p}$ (pe e) be the fiber of $\widetilde{N}(\tau)$ such that $p \in I_{p}$. Then, by [1, Lemma 5.8], we see that $I_{p} \cap \widetilde{\Delta}=p$. Hence $\underset{p \in \widetilde{e}}{U} I_{p}$ is a disk which is a disjoint union of the intervals. I . Since $\ell$ is carried by $\tau$ with all weights

[Figure 5]

positive, we have a rectangle $\widetilde{R}(\subset \widetilde{N}(\tau))$ such that one edge of $\widetilde{R}$ is $\widetilde{e}$, one edge of $\widetilde{R}$ is a subarc of a lift of $l$, and the rest edges are subarcs of two fibers of $\widetilde{N}(\tau)$. Since $\tilde{e}$ projects homeomorphically onto e, we see that $\widetilde{R}$ projects to the rectangle as in Assertion. This completes the proof of Assertion.

Let $F^{\prime}$ be the component of $F-l$ such that $F^{\prime} \supset m, F^{*}$ the surface obtained from $F^{\prime}$ by adding a boundary and $N\left(\subset F^{*}\right.$ ) the image of $N(\tau)$. Then we can reduce the subarcs of type $a_{1}$ in Figure 3 by an isotopy as in Figure 6. By Assertion, we can reduce [Figure 6] the subarcs of type $a_{2}$ in Figfure 3 by an ambient isotopy on $F^{*}$ as in Figure 7 .

\section{[Figure 7]}

It is easy to see that these operations terminates in finitely many steps and we see that $m$ is carried by $\tau$. 
This completes the proof of Lemma 2.3 .

Proof of Proposition 2.2. First we will show that $U_{1}$ actually exists. Then we will construct $U_{2}, U_{3}, \ldots$ inductively.

Assume that $U_{1}$ does not exist. Then there is a sequence of elements of $\varphi_{\ell},\left\{m_{i}\right\}_{i=1}^{\infty}$, such that $m_{j} \rightarrow L^{-}(i \rightarrow \infty)$. By [10, Chapitre 2, Proposition], there exists a maximal train track $\tau$ and a positive integer $n$ such that the stable lamination $L^{+}$is carried by $\tau$ with all weights positive and $f^{n}(\tau)$ is carried by $\tau$. If necessary, by taking $f^{n m}(\tau)$ for $\tau$, we may suppose that $\mathrm{B}_{\tau \mathrm{LL}^{-}}=\phi[10$, Chapitre 2$]$. Hence there exists a neighborhood of $L^{-}$ in $P L(F)$ which is disjoint from $B_{\tau}$. There is an integer $N$ such that $f^{N}(l) \subset$ Int $B_{\tau}$. On the other hand, since $f: P L(F) \rightarrow P L(F)$ is a continuous map, we have $f^{N}\left(m_{i}\right) \rightarrow L^{-}(i \rightarrow \infty)$, contradicting Lemma 2.3 . Hence $U_{1}$ exists.

Suppose that we have constructed $U_{1}, \ldots, U_{k-1}$. Let $\tau, n$ be as above. Then there exists a positive integer $N_{1}$ such that $\mathrm{f}^{\mathrm{nN}}\left(\mathrm{PL}(\mathrm{F})-\mathrm{U}_{\mathrm{k}-1}\right) \subset \mathrm{Int} \mathrm{B}_{\tau}$. Let $U_{k}=\mathrm{f}^{-\mathrm{nN}} \mathrm{N}_{1}\left(\mathrm{PL}(\mathrm{F})-\mathrm{B}_{\tau}\right)$. Since $\varphi(\ell ; k-1) \cap U_{k-1}=\phi$, we have $f^{n N_{1}}(\varphi(\ell ; k-1)) n f^{n N_{1}}\left(U_{k-1}\right)=$ $\left.\varphi\left(f^{\mathrm{nN}} 1(\ell) ; k-1\right)\right) \cap f^{\mathrm{nN}}{ }^{\left(U_{k-1}\right)}=\phi$. Hence $\varphi\left(f^{\mathrm{nN}} 1(\ell) ; k-1\right) \subset \ln t \mathrm{~B}_{\tau}$. Then, by Lemma 2.3 , we have $\varphi\left(f^{n N} 1(\ell) ; k\right) \subset B_{\tau}$. Then we have $U_{k} n \varphi(\ell ; k)=\phi$. This completes the proof of Proposition 2.2. 


\section{Proof of Theorem 1}

In this section we will give a proof of Theorem 1 . Throughout this section, let $H, \ell$ be as in section 1 . The key of the proof is Lemma 3.2 which is an easy estimation of $h(l)$ and Lemma 3.4 .

Lemma 3.1. Suppose that $\mathrm{h}(\ell)>-1$. Then there exists an element $\ell^{\prime}$ of $\varphi_{\ell}$ such that $\mathrm{h}\left(\ell^{\cdot}\right)<\mathrm{h}(\ell)$.

Proof. There exists an essential surface $F$ properly embedded in $(H, \partial H-l)$ such that $-\chi(F)=h(l)$. Since every incompressible, a-incompressible surface in $H$ is a disk, there is a disk $D$ in $H$ such that Int $D \cap F=\phi, D \cap \partial H=\partial D \cap \partial H=\alpha$ an arc and $D \cap F=\partial D \cap F=\beta$ an arc such that $\partial \alpha=\partial \beta, \alpha \cup \beta=\partial D$. Let $F^{\prime}$ be the 2-manifold obtained from $F$ by performing a surgery along $D$ and $\ell \cdot$ a component of $\partial F$. Clearly $\ell \cdot \in \mathscr{\varphi}_{\ell}$. By moving $\ell$ by a $t$ iny isotopy, we may suppose that $\ell^{\prime} \cap \partial F^{\prime}=\phi$. Since $F$ is essential, we see that a component of $F^{\prime}$, say $F^{\prime}$, is essential. And $\chi\left(F^{\prime}\right)>\chi(F)$. Hence we have $h\left(l^{\prime}\right)<h(l)$. This completes the proof of Lemma 3.1 .

As an immediate consequence of Lemma 3.1 , we have;

Lemma 3.2. Assume that $\varphi(l ; n)$ does not contain a simple loop of height -1 , i.e. if $\mathrm{m} \in \mathscr{Y}(\ell ; \mathrm{n})$, then $\partial \mathrm{H}-\mathrm{m}$ is incompressible in H. Then $\mathrm{h}(\mathrm{l})>\mathrm{n}$.

Lemma 3.3. If $\ell$ is of full type, then $h(\ell)>-1$, i.e. $2 \mathrm{H}-\ell$ 
is incompressible in $\mathrm{H}$.

For the definition of $\ell$ being of full type, see section 1 .

Proof. Assume that $\partial H-l$ is compressible in $H$. Then there is a compression disk D properly embedded in $(H, \partial H-l)$. Let $\left\{D_{1}, \ldots, D_{3 g-3}\right\}$ be a system of disks with respect to which $\ell$ is of full type. Then we may suppose that $\partial D_{i}, \partial D, \ell$ are geodesics for a fixed hyperbolic metric on $\partial H$. Assume that $\partial D=\partial D_{i}$ for some $i$. Then $\partial D$ intersects $l$, a contradiction. Hence we suppose that $\partial D \neq \partial D_{i}$ for each $i . B y$ cut and paste argument, we may suppose that $D$ intersects $U D_{i}$ in transverse arcs. Let $\Delta$ be an innermost disk in $D$, i.e. $\Delta \cap\left(U D_{i}\right)=\partial \Delta \cap\left(U D_{i}\right)=\alpha$, an arc and $\Delta \cap \partial H=\beta$, an arc such that $\alpha \cup B=\partial \Delta$. Let $P$ be the closure of the component of $H-N\left(U D_{i}\right)$ such that $\Delta \cap P \neq \varnothing$. Then $B \cap P$ is an arc and separates two boundary components of the pants $P \cap \partial H$. On the other hand, since $\ell$ is of full type, there is a subarc $\gamma$ of $\ell$ properly embedded in $P \cap \partial H$ such that $\gamma$ joins the two boundary components. Hence $\beta \cap \gamma \neq \varnothing$, a contradiction.

This completes the proof of Lemma 3.3 .

We say that a train track $\tau$ on $\partial H$ is of full type if there is a system of disks $D_{1}, \ldots, D_{3 g-3}$ in $H$ and a system of pants $P_{1}$,. $\ldots, \mathrm{P}_{2 \mathrm{~g}-2}$ as in section 1 which satisfies;

(i) $\tau$ and $\partial D_{1} \cup \ldots \cup \partial D_{3 g-3}$ intesect transversely and there is no 2-gon $B$ in $\partial H$ such that $\partial B=a U b$, where a is a differentiable arc on $\tau$, and $b$ is a subarc of $\partial D_{1} \cup \ldots U \partial D_{3 g-3}$ 
and

(ii) for each pair of boundary components of each pants $P_{i}$, there is a differentiable arc a on $\tau$ such that a is properly embedded in $P_{i}$, and a joins the boundary components.

Then, by Lemma 3.3 , we have;

Lemma 3.4. Suppose that the train track $\tau(\mathrm{C \partial H})$ is of full type. If $\ell$ is a simple loop which is carried by $\tau$ with all weights positive, then $\mathrm{h}(\mathrm{l})>-1$.

Proof of Theorem 1. Let $\mathrm{L}^{+}, \mathrm{L}^{-}$be the stable, and unstable laminations of f. By Proposition 2.2, there is a neighborhood $U$ of $L^{-}$in $P L(F)$ such that $U \cap \varphi(\ell ; n)=\varnothing$. Since $L^{+}$is of full type, there is a train track $\tau(C \partial H)$ which is of full type such that $L^{+}$ is carried by $\tau$ with all weights positive ([1, Lemma 5.2$])$. Let $N$ be a positive integer such that $f^{N}(P L(F)-U) \subset$ Int $B_{\tau}$. Then we have $f^{N}(\varphi(\ell ; n))=\varphi\left(f^{N}(l) ; n\right) \subset$ Int $B_{\tau}$. Hence, by Lemmas 3.2 and 3.4 , we see that $h\left(f^{N}(\ell)\right)>n$. Moreover, by $[10$, Chapitre 2], we may assume that $f\left(B_{\tau}\right) \subset I n t B_{\tau}$. Hence we see that if $m \geq N$, then $h\left(f^{m}(l)\right)>n$.

This completes the proof of Theorem 1 . 


\section{Proof of Theorem 2}

In this section we will prove Theorem 2 . First we will show that $M_{f}$ is well defined.

Let $H$ be a genus $g(>1)$ handlebody and $f: \partial H \rightarrow \partial H \quad a$ homeomorphism. Let $\left\{D_{1}, \ldots, D_{g}\right\},\left\{D_{1}, \ldots, D_{g}{ }^{\prime}\right\}$ be systems of mutually disjoint incompressible disks in $H$ such that UD (UD ${ }_{i}$ 'resp.) cuts $H$ into a 3 -cell. Let $M$ (M' resp.) be the 3-manifold obtained from $H$ by attaching 2-handles along the union of simple loops $U_{f}\left(\partial D_{i}\right)\left(U_{f}\left(\partial D_{i}{ }^{\prime}\right)\right.$ resp.).

Then we have;

Lemma 4.1. $M$ and $M$ ' are homeomorphic.

Proof. First we prepare a terminology. Let $B$ be a rectangle in $\partial H$ such that Int $B \cap\left(U \partial D_{i}\right)=\phi$, one edge of $B$ is a subarc $\alpha$ of $\partial D_{j}$, another edge of $\partial B$ is a subarc $B$ of $\partial D_{k}$, where $j \neq k$, with $B \cap\left(U D_{i}\right)=\alpha \cup B$. Then $D_{j} \cup B \cup D_{k}$ is a disk $D$ in $H$. By moving $D$ by a tiny isotopy, we may suppose that $D \cap \partial H=\partial D$ and $D \cap\left(U D_{i}\right)=\phi$. It is easy to see that the disks $\left\{D_{1}, \ldots, \hat{D}_{j}, \ldots, D_{g}, D\right\}$ cuts $H$ into a 3 -cell, where means removing the element. Then we say that $\left\{D_{1}, \ldots, D_{j}, \ldots, D_{g}, D\right\}$ is obtained from $\left\{D_{1}, \ldots, D_{g}\right\}$ by a band move. It is known that $\bigcup_{i} \underline{D}_{i}$ is ambient isotopic to the union of disks which is obtained from $\left\{D_{1}, \ldots . D_{g}\right\}$ by a sequence of band moves ([9,Korrolar 2]). Hence we may suppose that $\left\{D_{1}, \ldots . D_{g}{ }^{\prime}\right\}$ is obtained from $\left\{D_{1}, \ldots, D_{g}\right\}$ by a band move. We may suppose that $D_{i}=D_{i}{ }^{\prime}(2 \leq i \leq g)$, and $D_{1} \cap D_{1}{ }^{\prime}=\phi$. 
Then $\stackrel{M}{M}$ and $\stackrel{\dot{M}}{ }$ are homeomorphic to the manifold obtained from $H$ by attaching 2-handles along the union of simple loops $\partial D_{1} \cup \partial D_{1} \cdot U \partial D_{2} \cup \ldots U \partial D_{g}$, where $\stackrel{\leftrightarrow}{M}$ denotes the manifold obtained from $M$ by removing a 3 -cell from its interior. This shows that $M$ and $M$, are homeomorphic.

This completes the proof of Lemma 4.1 .

By Lemma 4.1, we may denote the manifold obtained as above $\bar{M}_{f}$. Then $M_{f}$ denotes the closed 3-manifold obtained from $\bar{M}_{f}$ by capping off the bondary by a 3-cell. It is easy to see that $H^{*}=c l\left(M_{f}-H^{\prime}\right)$ is a genus $g$ handlebody. Hence $\left(H_{,} H^{\prime} ; F\right)$, where $F=\partial H^{\prime}=\partial H^{\prime}$ $\left(\mathrm{CM}_{f}\right)$ is a Heegaard splitting of $M_{f} \cdot\left(H_{,} H^{\cdot} ; \mathrm{F}\right)$ is called a cannonical Heegaard splitting of $\mathrm{M}_{\mathrm{f}}$.

Then we will introduce the concept of rectangle condition of a Heegaard splitting by Casson-Gordon [2].

Let $S$ be the genus $g$ orientable surface and $P_{i}(i=1,2)$ be a pants (:disk with two holes) embedded in $S$, with $\partial P_{i}=$ $\ell_{1}{ }^{\mathrm{i}} \cup \ell_{2}{ }^{\mathrm{i}} \cup \ell_{3}{ }^{\mathrm{i}}$. We suppose that $\partial \mathrm{P}_{1}$ and $\partial \mathrm{P}_{2}$ intersect transversely. Let $Q_{1}, \ldots, Q_{m}$ be the compact surfaces obtained from $S-\left(\partial P_{1} \cup \partial P_{2}\right)$ by adding a boundary. We say that $P_{1}$ and $P_{2}$ are tight if;

(i) each $Q_{i}$ is not a 2-gon,

(ii) for each pair of, pair of boundary components, $\left(\left(\ell_{s}{ }^{1}, \ell_{t}{ }^{1}\right),\left(\ell_{p}{ }^{2}, l_{q}^{2}\right)\right)$ of $P_{1}$ and $P_{2}$ with $s \neq t, p \neq q$, there exists a rectangle $Q_{u}$ embedded in $P_{1}$ and $P_{2}$ such that Int $Q_{u} \cap\left(P_{1} \cup P_{2}\right)=\phi$, and the edges of $Q_{u}$ are subarcs of $\ell_{s}{ }^{1}$, $\ell_{t}^{1}, \ell_{p}^{2}$, and $\ell_{q}^{2}$.

Let $\ell_{1}, \ldots \ell_{3 g-3}\left(\ell_{1}, \ldots \ell_{3 g-3}\right.$, resp. $)$ be a system 
of mutually disjoint simple loops such that the closure of the complement of a regular neighborhood of $U \ell_{i}\left(U \ell_{i}\right.$ 'resp.) is a union of $2 \mathrm{~g}-2$ pants $\mathrm{P}_{1}, \ldots, \mathrm{P}_{2 \mathrm{~g}-2}\left(\mathrm{P}_{1}, \ldots, \mathrm{P}_{2 \mathrm{~g}-2}{ }^{\prime}\right.$ resp.). We say that $\left\{\ell_{1}, \ldots \ell_{3 g-3}\right\}$ and $\left\{\ell_{1}, \ldots \ell_{3 g-3}\right\}$ are tight if, for each pair $(i, j), P_{i}$ and $P_{j}$ are tight.

Let $\left(H, H^{\prime} ; \mathrm{F}\right)$ be a Heegaard splitting of a 3 -manifold $M$. We

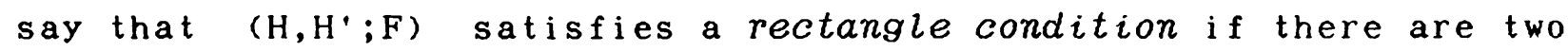
systems of mutually disjoint simple loops $\left\{\ell_{1}, \ldots, \ell_{3 g-3}\right\}$ and $\left\{\ell_{1}, \ldots\right.$ $\ldots, \ell_{3 g-3^{\prime}}$ which are tight and each $\ell_{i}\left(\ell_{i}{ }^{\prime}\right.$ resp.) is a boundary of a disk properly embedded in $H$ ( $H^{\prime}$ resp.).

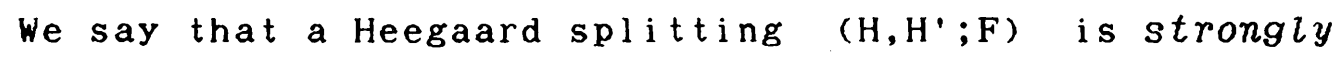
irreducible if there does not exist incompressible disks DCH, D'CH' such that $\partial D^{\prime} \partial D^{\prime}=\varnothing$.

Then Casson-Gordon proved;

Theorem [2]. If a Heegaard splitting (H,H';F) satisfies a rectangle condition, then $\left(\mathrm{H}^{\prime} \mathrm{H}^{\prime} ; \mathrm{F}\right)$ is strongly irreducible.

In this paper we will show;

Theorem 4.2. Let $H$ be as in section 1 and $\mathrm{f}: \partial \mathrm{H} \rightarrow \partial \mathrm{H}$ be $a$ pseudo-Anosov homeomorphism. Suppose that the invariant laminations of $\mathbf{f}$ are of full type. Then there is an integer $\mathrm{N}_{1}$ such that if $\mathrm{m}>\mathrm{N}_{1}$, then the cannonical Heegaard splitting of ${ }_{\mathrm{M}^{\mathrm{m}}}$ satisfies a rectangle condition. 
Proof. Let $\mathrm{L}^{+}\left(\mathrm{L}^{-}\right.$resp.) be the stable (unstable resp.) lamination of $f$. We suppose that $L^{+}\left(L^{-}\right.$resp.) is of full type with respect to a system of disks $\left\{D_{1}, \ldots, D_{3 g-3}\right\}$

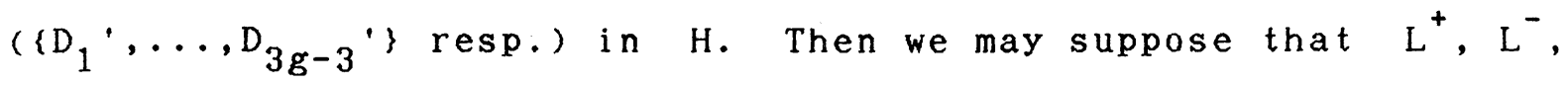
$U \partial D_{i}, U \partial D_{i}{ }^{\prime}$ are unions of geodesics on $\partial H$. The universal cover of $\partial H$ is isometric to the hyperbolic plane $\mathbb{H}^{2}$. Let $\tilde{f}: \mathbb{H}^{2} \rightarrow \mathbb{H}^{2}$ be a lift of $f$, and $S_{\infty}^{1}$ be the circle at $\infty$ of $\mathbb{H}^{2}$. Then it is known that $\tilde{f}$ has a unique continuous extension to $\mathbb{H}^{2} \cup S_{\infty}^{1}=D^{2}$ ([1, Lemma 3.7$])$. Moreover, there is a positive integer $n$ such that if $g: D^{2} \rightarrow D^{2}$ is the extension of a lift of $f^{n}$ then $\left.g\right|_{S_{\infty}}$ has finitely many fixed pionts $A_{1}, \ldots, A_{2 n} \quad(n \geq 1)$ on $S_{\infty}^{1}$, alternately contracting and expanding. We suppose that $A_{1}, \ldots, A_{2 n}$ are on $S_{\infty}^{1}$ in this order, $A_{1}, \ldots, A_{2 n-1}$ are contracting and $A_{2}, \ldots, A_{2 n}$ are expanding. Let $I_{j}(j=1, \ldots, 2 n-1)$ be a closed interval in $S_{\infty}^{1}$ bounded by $A_{j}$ and $A_{j+1}$ such that Int $I_{j} \cap\left(U A_{i}\right)=\phi$, and $I_{2 n}=c \ell\left(S_{\infty}^{1}{ }_{j=1}^{2 n-1} I_{j}\right)$. Let $\tilde{L}^{+}\left(\tilde{L}^{-}\right.$resp. $)\left(C H^{2}\right)$ be the lift of $L^{+}$ ( $\mathrm{L}^{-}$resp.), and $\gamma_{1}\left(\delta_{1}\right.$ resp.) be the geodesics of $\tilde{L}^{+}\left(\tilde{L}^{-}\right.$resp.) which joins $A_{1}$ and $A_{3}\left(A_{2}\right.$ and $A_{4}$ resp.).

Then we will show;

Assertion. For each $\partial D_{i}\left(\partial D_{i}\right.$ ' resp.), there exists a lift e $\left(\subset H^{2}\right)$ of it such that the endpoints of $e$ are contained in $I_{1}$ and $I_{2}\left(I_{1}\right.$ and $I_{2 n}$ resp.).

Proof. Since each leaf of $\mathrm{L}^{+}$is dense in $\mathrm{L}^{+}$ ( $[1$, Theorem 4.8$])$, there is a sequence of lifts of the points of 
$L^{+} \cap \partial D_{i},\left\{a_{i}\right\}_{i=1}^{\infty}$ such that $a_{i} \in \delta_{1}$, and $a_{i} \rightarrow A_{2}(i \rightarrow \infty)$. Let $\left\{\varepsilon_{i}\right\}_{i=1}^{\infty}$ be the sequence of the lifts of $\partial D_{i}$ such that $\varepsilon_{i} \cap \delta_{1}=a_{i}$. Since each $\varepsilon_{i}$ is a geodesic, we see that at least one endpoint of $\varepsilon_{i}$ is contained in $I_{1}$ or $I_{2}$ for sufficiently large i. Assume that the other endpoints of $\varepsilon_{i}$ are not contained in $I_{2}$ or $I_{1}$. Then we have $\lim _{\mathrm{i}} \arg \left(\varepsilon_{i}, \delta_{1}\right)=0$, where $\arg \left(\varepsilon_{i}, \delta_{1}\right)$ denotes the angle between the two geodesics. Since $\mathrm{L}^{+}$is a closed set, we have $\partial \mathrm{D}_{\mathrm{i}} \subset \mathrm{L}^{+}, \mathrm{a}$ contradisction.

This completes the proof of Assertion.

Let $P_{1}, \ldots, P_{2 g-2}\left(P_{1}, \ldots, P_{2 g-2}\right.$, resp. $)$ be a system of

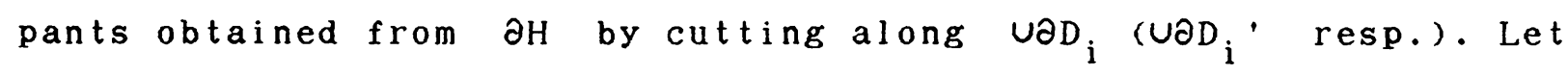
$\partial D_{\alpha}, \partial D_{\beta}$ and $\partial D_{\gamma}, \partial D_{\delta}$, be pairs of boundary components of a pair $\left(\mathrm{P}_{i}, \mathrm{P}_{\mathrm{j}}^{\prime}\right)$. By Assertion, there are lifts $\tilde{\alpha}, \tilde{\beta}(\tilde{\gamma}, \tilde{\delta}$ resp. $)$ of $\partial D_{\alpha}, \partial D_{B}\left(\partial D_{\gamma}, \partial D_{\delta}\right.$, resp. $)$ in $\mathbb{H}^{2}$ such that $\tilde{\alpha} \cap \gamma_{1} \neq \phi, \tilde{\beta} \cap \gamma_{1} \neq \phi$, $\tilde{\gamma} \cap \delta_{1} \neq \phi, \tilde{\delta} \cap \delta_{1} \neq \phi$, the endpoints of $\tilde{\alpha}, \tilde{\beta}(\tilde{\gamma}, \tilde{\delta}$ resp.) are contained in $I_{1}$ and $I_{2}$ ( $I_{1}$ and $I_{2 n-1}$ resp.), and the subarc of $\gamma_{1}\left(\delta_{1}\right.$ resp.) bounded by $\tilde{\alpha} \cap \gamma_{1}, \tilde{\beta} \cap \gamma_{1}\left(\tilde{\gamma} \cap \delta_{1}, \tilde{\delta} \cap \delta_{1}\right.$ resp. $)$ projects to an arc properly embedded in $P_{i}\left(P_{j}{ }^{\prime}\right.$ resp.). Then there is a constant $N_{i} \underset{\sim}{j}, \alpha, \beta, \underset{\sim}{\gamma}, \delta$ such that if $m>N_{i}, j, \alpha, \beta, \gamma, \delta$, then the endpoints of $\tilde{f}^{\mathrm{m}}(\tilde{\alpha})$ and $\tilde{f}^{m}(\tilde{\beta})$ are separated by the endpoints of $\tilde{\gamma}$ and $\tilde{\delta}$. Let $N_{1}=\max \left\{N_{i, j, \alpha, \beta, \gamma, \delta} \mid 1 \leq \mathrm{i}, \mathrm{j} \leq 2 \mathrm{~g}-2, \alpha, \beta, \gamma, \delta\right\}$. Then $N_{1}$ satisfies the conclusion of Theorem 4.2.

This completes the proof of Theorem 4.2.

Lemma 4.3. Suppose that a Heegaard splitting $\left(\mathrm{H}, \mathrm{H}^{\prime} ; \mathrm{F}\right)$ of $\mathrm{M}$ is strongly irreducibe and that M contains a 2-sided incompressible 
surface $\mathrm{S}$. Then there is a surface $\mathrm{S}^{\prime}$ which is ambient isotopic to $\mathrm{s}$ and satisfies;

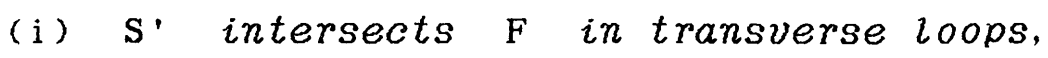

(i i) every component of $\mathrm{S}^{\cdot} \cap \mathrm{H}$ is not a disk, and

(iii) just one component of $\mathrm{S}^{\prime} \cap^{\mathrm{H}^{\prime}}$ is a disk.

Proof. This lemma is proved by using the argument of [6, Chapter II], which was used to prove the Haken's theorem. We assume that the reader is familiar with the proof. We note that Jaco considered the case of $S$ being the 2-sphere, but the argument works

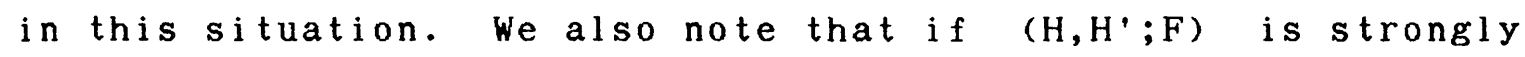
irreducible, then $M$ is irreducible ([3, Theorem 2.1]).

We may suppose that each component of $S \cap H$ is a disk and the number of the component is minimal among all surfaces which are ambient isotopic to $S$. By the minimality, we see that $S \cap H^{\prime}$ is an incompressible surface in $H^{\prime}$. Hence we have a hierarchy for $\mathrm{S} \cap \mathrm{H}^{\prime},\left(\mathrm{S}_{2}{ }^{(0)}, \alpha_{0}\right),\left(\mathrm{S}_{2}{ }^{(1)}, \alpha_{1}\right), \ldots,\left(\mathrm{S}_{2}{ }^{(\mathrm{p})}, \alpha_{\mathrm{p}}\right)$, and a sequence of isotopies of type $A$ in $M$ which realizes the hierarchy. Let $S(q)$ $(0 \leq q \leq p+1)$ be the image of $S$ after the $q-t h$ isotopy of type $A$, i.e. $S^{(q)} \cap H^{\prime}=S_{2}{ }^{(q)}$. Let $k$ be the integer such that $S_{2}{ }^{(k)}$ does not contain a disk and $S_{2}{ }^{(k+1)}$ contains a disk. We note that $S_{2}{ }^{(k+1)}$ contains just one disk. Then, by Theorem 4.2 and a theorem of Casson-Gordon, we see that each component of $S^{(k+1)} \cap H$ is not a disk. Hence $S^{\prime}=S^{(k+1)}$ satisfies the the conclusions of Lemma 4.3 . This completes the proof of Lemma 4.3 .

Lemma 4.4. Let $\mathrm{f}: \partial \mathrm{H} \rightarrow \partial \mathrm{H}$ be a pseudo-Anosov homeomorphism. 
Suppose that the stable lamination $\mathrm{L}^{+}$of $\mathrm{f}$ is of full type. Then, for each $\mathrm{n}(2-1)$, there exists a neighborhood $\mathrm{U}$ of $\mathrm{L}^{+}$in $\mathrm{PL}(\partial \mathrm{H})$ such that the height of every simple loop contained in $\mathrm{U}$ is greater than $\mathrm{n}$.

Proof. Assume that the conclusion of Lemma 4.3 does not hold. Then there exists an integer $n(2-1)$, and a sequence of simple loops $\left\{\ell_{i}\right\}_{i=1}^{\infty}$ on $\partial H$ such that $\ell_{i} \rightarrow L^{+}(i \rightarrow \infty)$, and $h\left(l_{i}\right) \leq n$. By $[10]$, there is a maximal train track $\tau$ on $\partial H$ and a positive integer $m$ such that $L^{+}$is carried by $\tau$ with all weights positive and $f^{m}(\tau)$ is carried by $\tau$. Set $B_{i}=B_{f}^{m i}(\tau)(i \geq 1)$. Then, by $[10]$, we see that $B_{p+1} \subset$ Int $B_{p}$, and $B_{p}$ converges to $L^{+}$. If necessary, by taking a subsequence of $\left\{\ell_{i}\right\}$, we may suppose that $\ell_{i} \subset$ Int $B_{i}(i \geq 1)$. By Lemmas 3.1 and 2.2 , we have a sequence of simple loops $\left\{\ell_{i}\right\}_{i=1}^{\infty}$ such that $h\left(l_{i}{ }^{\prime}\right) \leq n-1, \ell_{i}{ }^{\prime} \in B_{i}$, hence, $\ell_{i} \rightarrow^{\prime} L^{+}(i \rightarrow \infty)$. By applying this argument finitely many times, we get a sequence of simple loops $\left\{\ell_{i}{ }^{*}\right\}_{i=1}^{\infty}$ such that $h\left(\ell_{i}{ }^{*}\right)=-1$ and $\ell_{i}^{*} \rightarrow L^{+}(i \rightarrow \infty)$. But this contradicts Lemma 3.4 .

This completes the proof of Lemma 4.4 .

Proof of Theorem 2. By Lemma 4.4, there is a neighborhood $U^{+}$ (U $U^{-}$resp.) of $\mathrm{L}^{+}\left(\mathrm{L}^{-}\right.$resp.) such that the height of every simple loop in $U^{+}$( $U^{-}$resp.) is greater than $2 n+1$ (1 resp.). Let $N^{\prime}$ be an integer such that $f^{m}\left(P L(\partial H)-U^{-}\right) \subset U^{+}$for each $m\left(>N^{\prime}\right)$. And let $N=\max \left\{N^{\prime}, N_{1}\right\}$, where $N_{1}$ is the constant obtained in Theorem 4.2. Then we will see that this $N$ satisfies the conclusion of Theorem 2 . 
Assume that there is an integer $m(>N)$ such that $M_{f}$ contains an incompressible surface $S$ whose genus is less than or equal to $n$, i.e. $\chi(S) \geq 2-2 n$. We may suppose that $S$ satisfies the conclusions of Lemma 4.2. Let $\mathrm{S}_{1}=\mathrm{S} \cap \mathrm{H}$ and $\mathrm{S}_{2}=\mathrm{S} \cap \mathrm{H}^{\prime}$. Then $x(S)=x\left(S_{1}\right)+\chi\left(S_{2}\right) 22-2 n$. Let $D$ be the disk component of $S_{2}$. Then $D$ is the only component which makes a positive contribution in $\chi\left(S_{1}\right)+\chi\left(S_{2}\right)$. Hence we have $x\left(S_{1}\right)+\chi\left(S_{2}\right) \leq x\left(S_{2}\right)+1$. Then $2-2 n$ $\leq \chi\left(S_{2}\right)+1$ and $\chi\left(S_{2}\right) \geq 1-2 n$. Let $s^{*}$ be a component of $S_{2}$. Then $x\left(S^{*}\right) \geq 1-2 n$. Hence $f^{m}(\partial D) \subsetneq U^{+}$, contradicting the fact that $\partial D$ does not contained in $U^{-}$.

This completes the proof of Theorem 2 . 


\section{Examples}

In this section we will give ways of constructing pseudo-Anosov homeomorphisms which satisfies the assumptions of Theorems 1 and 2 . Throughout this section let $H$ be as in section 1 and $T_{\ell}$ the Dehn twist along the simple loop $\ell$ in $\partial H$.

Example 1. Let $\ell(C \partial H)$ be a simple loop which is of full type and $m(C \partial H)$ a simple loop such that $\ell \cup m$ fills up $\partial H$, i.e. the number of intersection of $l$ and $m$ is minimal among all simple loops which are isotopic to $m$ and each component of $\partial H-(l U m)$ is an open disk. Then, by [4,Exposé 13], we see that $T_{m}{ }^{\circ} T_{\ell}^{-n}$ is isotopic to a pseudo-Anosov homeomorphism for each positive integer $n$ and the stable lamination tends to $l$ if $n$ grows larger. Hence $\mathrm{T}_{\mathrm{m}} \circ \mathrm{T}_{\ell}^{-n}$ satisfies the assumption of Theorem 1 if $n$ is sufficiently large.

Example 2. Let $\varphi$ be a pseudo-Anosov homeomorphism such that $\varphi^{-1}$ satisfies the assumption of Theorem 1 , i.e. the unstable lamination $L^{-}$of $\varphi$ is of full type. Let $D$ be a component of the system of disks with respect to which $L^{-}$is of full type. Then, as observed in the proof of [9, Lemma 2.5$], \varphi^{-\mathrm{k}} \circ \mathrm{T} \partial D^{\circ} \varphi^{\mathrm{k}} \circ \mathrm{T}_{\partial D}{ }^{-1}$ is isotopic to a pseudo-Anosov homeomorphism for a sufficiently large k, and the invariant laminations tend to $L^{-}$and $T_{\partial D}\left(L^{-}\right)$if $k$ grows

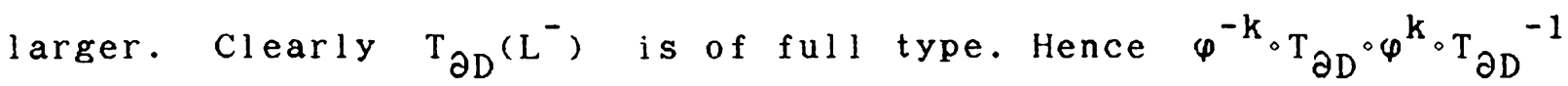
satisfies the assuption of Theorem 2 if $k$ is sufficiently large. 


\section{References}

[1] A. J. Casson, Automorphisms of surfaces after Nielsen and Thurston, Lecture note, University of Texas at Austin [2] A. J. Casson, and C. McA Gordon, Manifolds with irreducible Heegaard splittings of arbitrarily high genus, to appear [3] , Reducing Heegaard splittings,

preprint

[4] A. Fathi, and F. Laudenbach, Diffeomorphismes pseudo-Anosov et dècompositions de Heegaard, C. R. Acad. Sc. Paris, 291(1980) A423 [5] A. Fathi, F. Laudenbach, and V. Poenaru, Travaux de Thurston sur les surfaces, Astèrisque, 66-67(1979)

[6] J. Hempel, 3-manifolds, Ann. of Math. Studies 86(1976), Princeton University Press, Princeton N.J.

[7] W. Jaco, Lectures on three-manifold topology, CBMS Regional Conference Series in Math., 43(1980)

[8] T. Kobayashi, Structures of full Haken manifolds, Osaka J. Math. ( to appear)

[9] D. D. Long, A note on the normal subgroups of mapping class groups, Math. Proc. Camb. Phil. Soc. 99(1986), 79-87

[10] A. Papadopoulos, Reseaux ferroviaires, diffeomorphismes pseudo-Anosov et automorphismes symplectiques de l'homologie d'une surface, publications mathematiques d'orsay (1983)

[11] H. Zieschang, Uber einfache Kurven auf Vollbrezeln, Abh. Math. Sem. Univ. Hamburg 25(1962), 231-250 

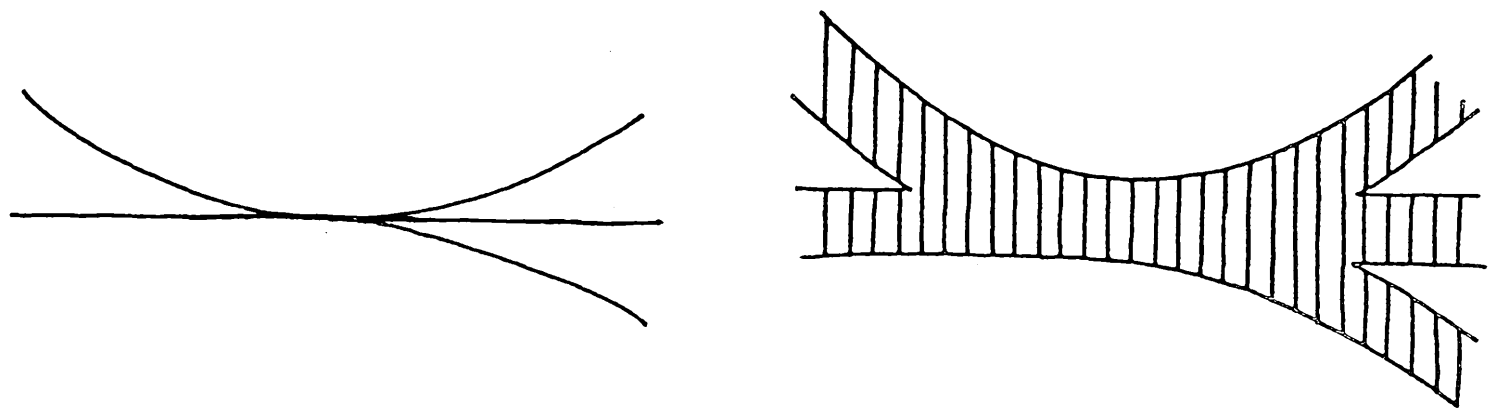

Figure 1 

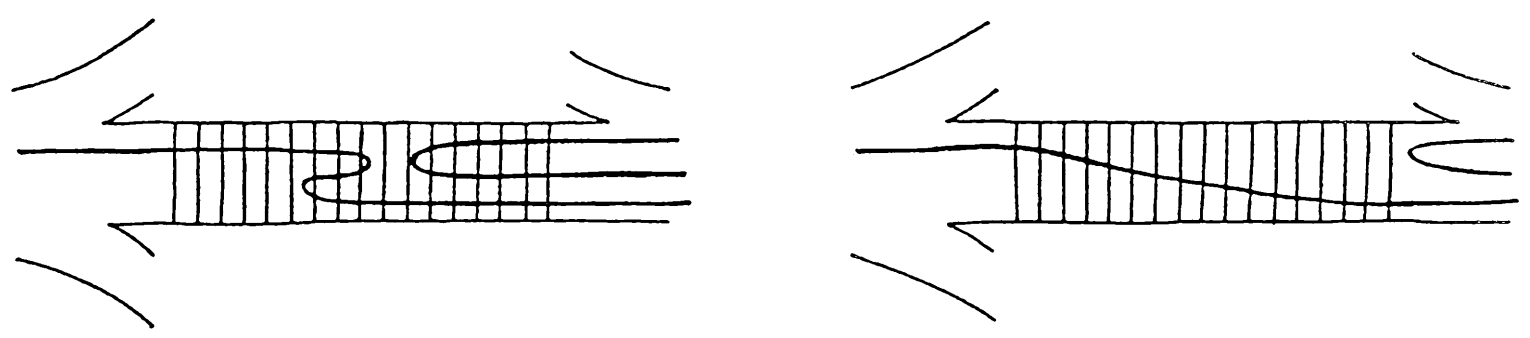

Figure 2

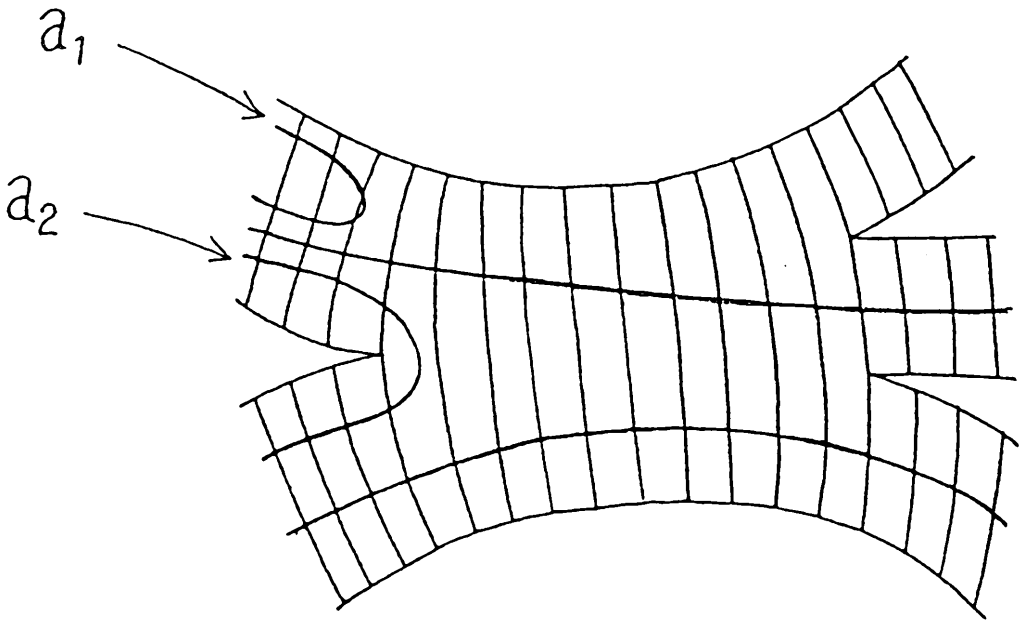

Figure 3 
$19 \%$

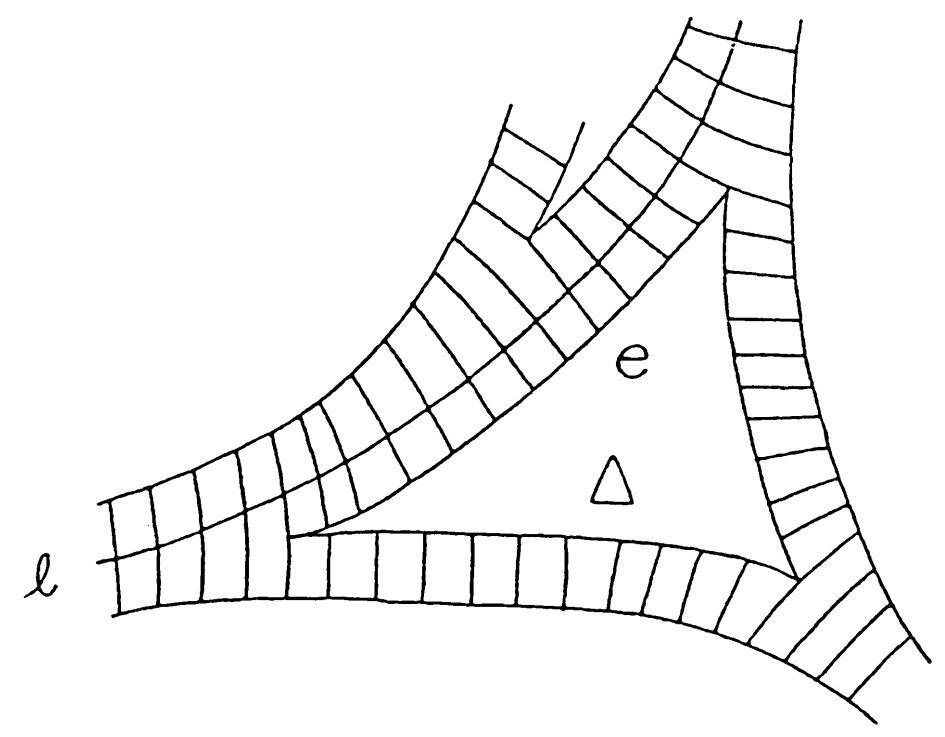

Figure 4

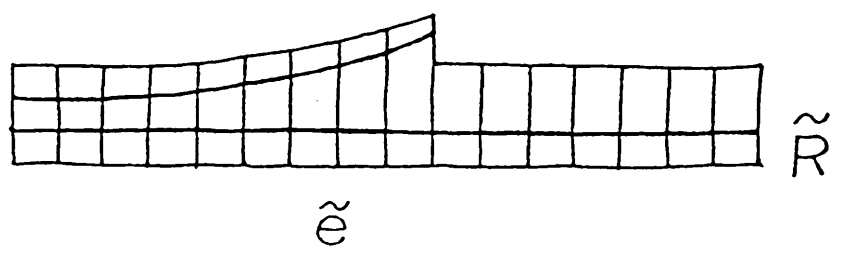

Figure 5 


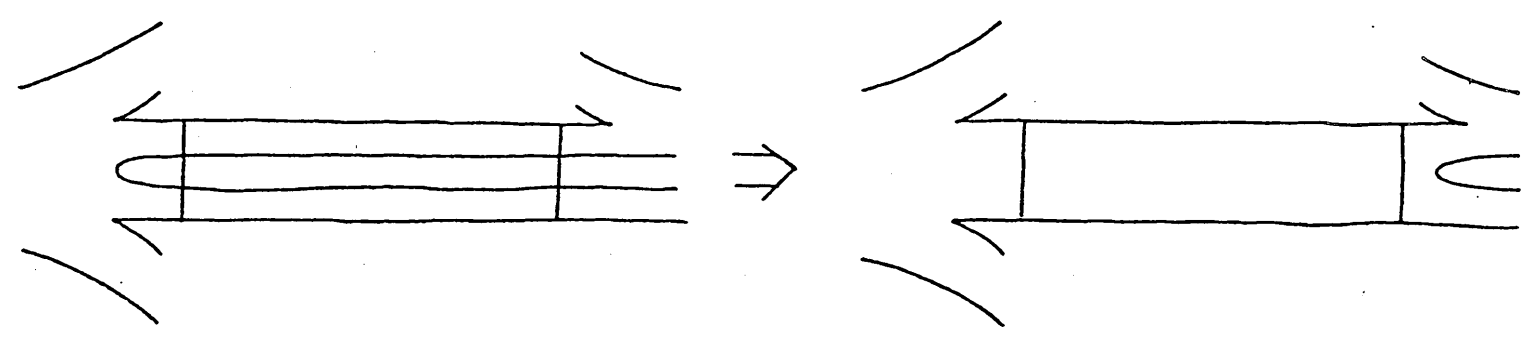

Figure 6
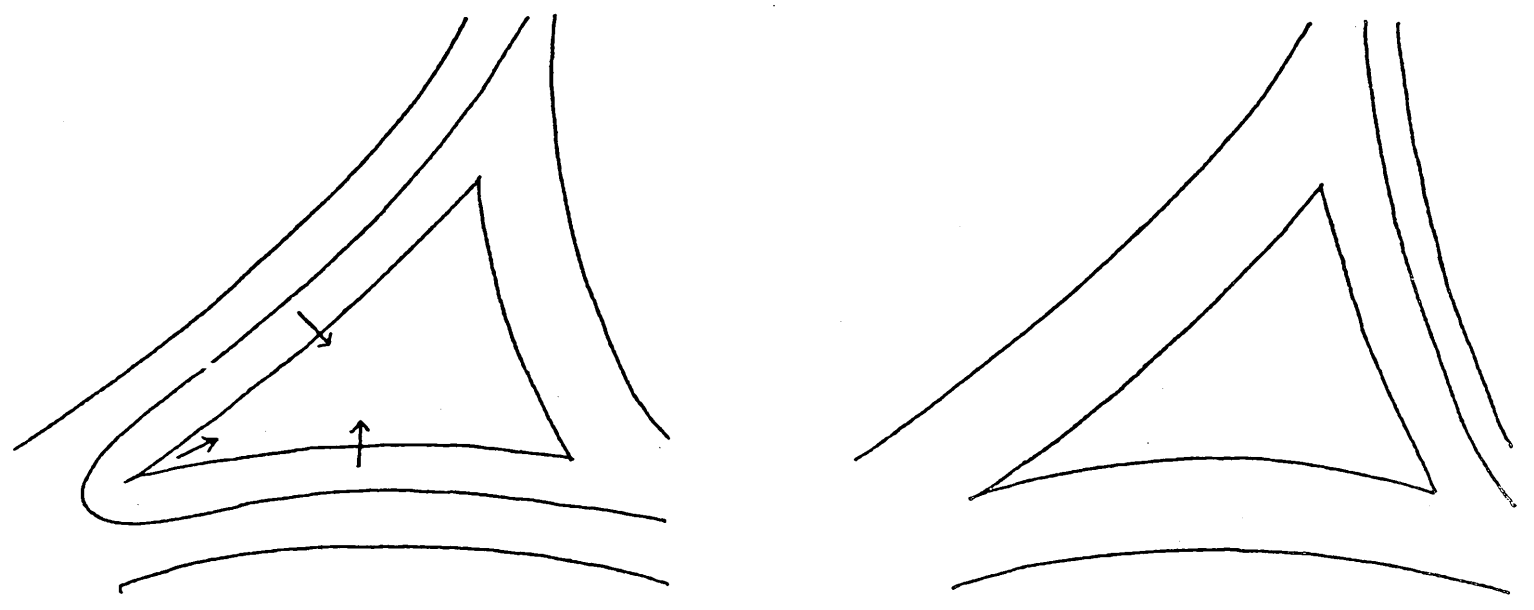

Figure? 